\title{
Prevalence and Characteristics of Salmonella Isolated from Free-Range Chickens in Shandong Province, China
}

\author{
Xiaonan Zhao, ${ }^{1}$ Yanxia Gao, ${ }^{2}$ Chaoqun Ye, ${ }^{1}$ Lingling Yang, \\ Tao Wang, ${ }^{1}$ and Weishan Chang ${ }^{1}$ \\ ${ }^{1}$ College of Animal Science and Technology, Shandong Agricultural University, Taian 271000, China \\ ${ }^{2}$ College of Life Sciences, Taishan Medical University, Taian 271000, China
}

Correspondence should be addressed to Xiaonan Zhao; zhaoxn1214@126.com and Weishan Chang; sdwschang@sina.com Received 7 May 2016; Accepted 24 August 2016

Academic Editor: Yi-Ping Liu

Copyright (C) 2016 Xiaonan Zhao et al. This is an open access article distributed under the Creative Commons Attribution License, which permits unrestricted use, distribution, and reproduction in any medium, provided the original work is properly cited.

Compared with chickens raised in intensively managed breeding farms, free-range chickens in China are quite popular due to lower breeding density and less antibiotics usage. However, investigations about Salmonella enterica from free-range chickens are quite rare. The aim of the present study was to investigate prevalence and characteristics of Salmonella in free-range chickens in Shandong province, China. During the period of August and November 2015, 300 fresh fecal swabs from different broilers in three free-range chicken farms (100 samples per farm) were collected to isolate Salmonella, and then these isolates were subjected to serotyping, antibiotic sensitivity testing, enterobacterial repetitive intergenic consensus-polymerase chain reaction (ERIC-PCR), and multilocus sequence typing (ST). A total of 38 Salmonella isolates $(38 / 300,12.7 \%)$ were recovered. The most common serotype was Enteritidis (81.6\%), followed by Indiana (13.2\%) and Typhimurium (5.3\%). Twenty-two out of 38 isolates (57.9\%) were resistant to ampicillin, the highest resistance rate, but resistance rates to cefazolin, cefotaxime, and ceftazidime were only $7.9 \%$. The multidrug resistance (MDR) rate was 26.3\%. Additionally, the Salmonella isolates could be classified into 25 genotypes by ERIC-PCR and were divided into three ST types (ST11, ST17, and ST19), with ST11 the highest isolation rate (81.6\%). In summary, as with other poultry, free-ranging chickens may also serve as potential reservoir for antibiotic resistant Salmonella, thereby posing a threat to public health.

\section{Introduction}

Salmonella enterica is one of the most important pathogenic bacterial causes of food-borne diseases [1]. At present, more than 2,600 serotypes were identified, and most serotypes can cause food-borne infection [2]. Salmonella infections can result in gastrointestinal problems such as gastroenteritis, typhoid fever, and paratyphoid fever or even cause death in serious infections, especially for younger people and the elderly [3-5]. In China, bacterial food-borne disease cases are frequently caused by Salmonella, and Salmonella in foodproducing animals is a threat to public health $[6,7]$. Foodproducing animals especially chickens and pigs are regarded as the most important Salmonella carriers. Epidemiological investigations of Salmonella from chickens, pigs, and their meat products have shown that drug resistance of Salmonella has been increasing in China and other countries [7-10].
In recent years, free-range chicken farms are major contributors to organic food production in Shandong province, China. The free-range chicken farms are divided by spatially separated (chickens and crops are physically isolated), rotational (chickens can roam into field only after crops are harvested), and fully combined (chickens and crops are allowed to interact freely) farming. Importantly, although the chickens are purchased from local commercial poultry hatchery, fewer antibiotics are used for therapy or growth promotion. Compared with chickens raised in intensively managed breeding farms, free-range chickens in China are quite popular due to lower breeding density and less antibiotics usage.

However, investigations about Salmonella from freeranging chickens are quite rare in China. Therefore, the aim of the present study was to isolate Salmonella from freeranging chickens in Shandong province, China, and then 
these isolates were subjected to serotyping, antibiotic sensitivity testing, enterobacterial repetitive intergenic consensuspolymerase chain reaction (ERIC-PCR), and multilocus sequence typing (ST).

\section{Materials and Methods}

2.1. Sample Collection and Process. Between August and November 2015, 300 fresh fecal swabs of chickens aged about 6 weeks (100 samples per farm) were obtained from three free-range chicken farms (Jinan, Linyi, and Laiwu) (about 500 chickens per farm) in Shandong province, China. Fresh fecal samples were collected from near the chickens using sterile cotton swabs. After sampling, the swabs were transported to our lab in an ice box and processed within $6 \mathrm{~h}$. The cotton swabs were immediately put into vials containing $100 \mathrm{~mL}$ of germ-free BPW media and shaken sufficiently at $37^{\circ} \mathrm{C}$ for 18 to $20 \mathrm{~h}$. Then $0.5 \mathrm{~mL}$ and $0.1 \mathrm{~mL}$ of the enrichment broth were added to $10 \mathrm{~mL}$ of Tetrathionate Broth (TT, BectonDickinson, USA) and Rappaport-Vassiliadis Medium (RV, Becton-Dickinson, USA), respectively, at $42^{\circ} \mathrm{C} \pm 1,100 \mathrm{ppm}$ for 22 to $24 \mathrm{~h}$. Then, the bacteria in TT and RV were inoculated to xylose lysine tergitol 4 (XLT4, Becton-Dickinson, USA) agar and incubated at $37^{\circ} \mathrm{C} \pm 1$ overnight. Suspected Salmonella colonies were then inoculated to trisaccharide agar slant and incubated at $35^{\circ} \mathrm{C} \pm 1$ for $24 \mathrm{~h}$. The typical Salmonella colonies were identified by the VITEK system (BioMerieux, Marcy l'Etoile, France) and then verified by PCR amplification of inherent gene invA [11].

2.2. Salmonella Serotyping. Commercial serodiagnosis kits for Salmonella (Ningbo Tianrun Bio-pharmaceutical Co., Ltd., China) were used to carry out the plate agglutination tests and identify serotypes of Salmonella. The serotyping scheme was referenced to the Kauffman-White salmonella serotyping scheme [12].

2.3. Antimicrobial Susceptibility Testing. A panel of 14 antibiotics, amikacin (AMK), gentamicin (GEN), kanamycin (K), norfloxacin (NOR), ampicillin (AMP), cefazolin (CFZ), cephradine (RAD), cefotaxime (CTX), chloramphenicol (CHL), tetracycline (TET), co-trimoxazole (SXT), ceftazidime (CAZ), ciprofloxacin (CIP), and doxycycline (DOX), was used to test the antimicrobial susceptibility of Salmonella by Kirby-Bauer disk diffusion method, as recommended by the Clinical and Laboratory Standards Institute [13]. An isolate was considered as multidrug-resistant (MDR) when exhibiting resistance to antimicrobials of at least three different classes [14]. Escherichia coli ATCC 25922 and Pseudomonas aeruginosa ATCC 27853 were used as quality control strains.

2.4. ERIC-PCR. The primers ERIC 1 ( $5^{\prime}$-ATGTAAGCTCCTGGGATTCAC-3') and ERIC 2 ( $5^{\prime}$-AAGTAAGTGACTGGGGTGAGCG-3') were synthesized by Takara Biotechnology Co., Ltd. (Dalian, China). The total volume of PCR reaction was $25 \mu \mathrm{L}$, including $10 \mathrm{x}$ buffer $2.5 \mu \mathrm{L}, 2.5 \mathrm{mmol} / \mathrm{L}$ dNTP $2.0 \mu \mathrm{L}$, forward and reverse primers $(20 \mathrm{pmol} / \mathrm{L}) 1 \mu \mathrm{L}$ for each, $25 \mathrm{mmol} / \mathrm{L} \mathrm{MgCl}_{2} 1.5 \mu \mathrm{L}$, Taq polymerase $5 \mathrm{U}$, and
TABLE 1: Serotyping of Salmonella isolated from three free-range chicken farms.

\begin{tabular}{lcccc}
\hline \multirow{2}{*}{ Serovar } & \multicolumn{3}{c}{ Number of isolates } & \\
& Jinan & Linyi & Laiwu & Total $(n=38)$ \\
Enteritidis & $8(80 \%)$ & $9(69.2 \%)$ & $\begin{array}{c}14 \\
(n=13)\end{array}$ & $31(81.6 \%)$ \\
Indiana & $2(20 \%)$ & $3(23.0 \%)$ & 0 & $5(13.2 \%)$ \\
Typhimurium & 0 & $1(7.7 \%)$ & $1(6.7 \%)$ & $2(5.3 \%)$ \\
\hline
\end{tabular}

genome DNA $1.5 \mu \mathrm{L}$. The ERIC-PCR was completed by an initial heat activation of $10 \mathrm{~min}$ at $94^{\circ} \mathrm{C}$, then 31 cycles of $30 \mathrm{~s}$ at $92^{\circ} \mathrm{C}$ and $60 \mathrm{~s}$ at $40^{\circ} \mathrm{C}, 8 \mathrm{~min}$ at $65^{\circ} \mathrm{C}$, and an extension of $8 \mathrm{~min}$ at $65^{\circ} \mathrm{C}$. PCR products were run on an agarose gel electrophoresis (2\%) at $100 \mathrm{~V}$ for $1 \mathrm{~h} \mathrm{[15].}$

2.5. ERIC-PCR Fingerprint Analysis. The samples that could be amplified by ERIC-PCR were marked as "1"; otherwise, they were marked as "0." They were submitted to Gel Image System (Version 4.00) to give a matrix graph automatically. The unweighted pair group method using averages algorithm (UPGMA) was used to obtain the clustering dendrograms (NTSYS-pc 2.10 software). The individual isolates were regarded as an independent operational taxonomic unit (OUT), and isolates with a similarity over $90 \%$ were assumed to be of the same origin [15].

2.6. MLST. The seven pairs of housekeeping genes for Salmonella MLST assays from University College Cork (http://mlst.ucc.ie/), aroC, dnaN, hemD, hisD, pure, sucA, and thrA, were used as indexes for gene classification. Finally, these results were compared to the S. enterica MLST database (http://mlst.warwick.ac.uk/mlst/dbs/Senterica) for the ST types [16].

\section{Results}

3.1. Isolation of Salmonella. In the present study, 38 Salmonella isolates were obtained from 300 samples from three free-range chicken farms, including 10 isolates (numbers 110) from Jinan (10/100, 10\%), 13 (numbers 11-23) from Linyi $(13 / 100,13 \%)$, and 15 (numbers $24-38$ ) from Laiwu (15/100, $15 \%)$.

3.2. Serotypes of Salmonella. The 38 Salmonella isolates were classified into three serotypes according to the plate agglutination tests. The most common serotype was S. enterica serovar Enteritidis $(n=31,81.6 \%)$, followed by S. enterica serovar Indiana $(n=5,13.2 \%)$ and S. enterica serovar Typhimurium $(n=2,5.3 \%)$ (Table 1$)$.

3.3. Antimicrobial Susceptibility Testing. Drug resistance rates for the 38 Salmonella isolates (Table 3) were as follows: ampicillin, 57.9\% (22/38); co-trimoxazole and kanamycin, $29.0 \%$ each (11/38); tetracycline, $26.3 \%$ (10/38); doxycycline and gentamicin, $23.7 \%$ each (9/38); and cefazolin, cefotaxime, 


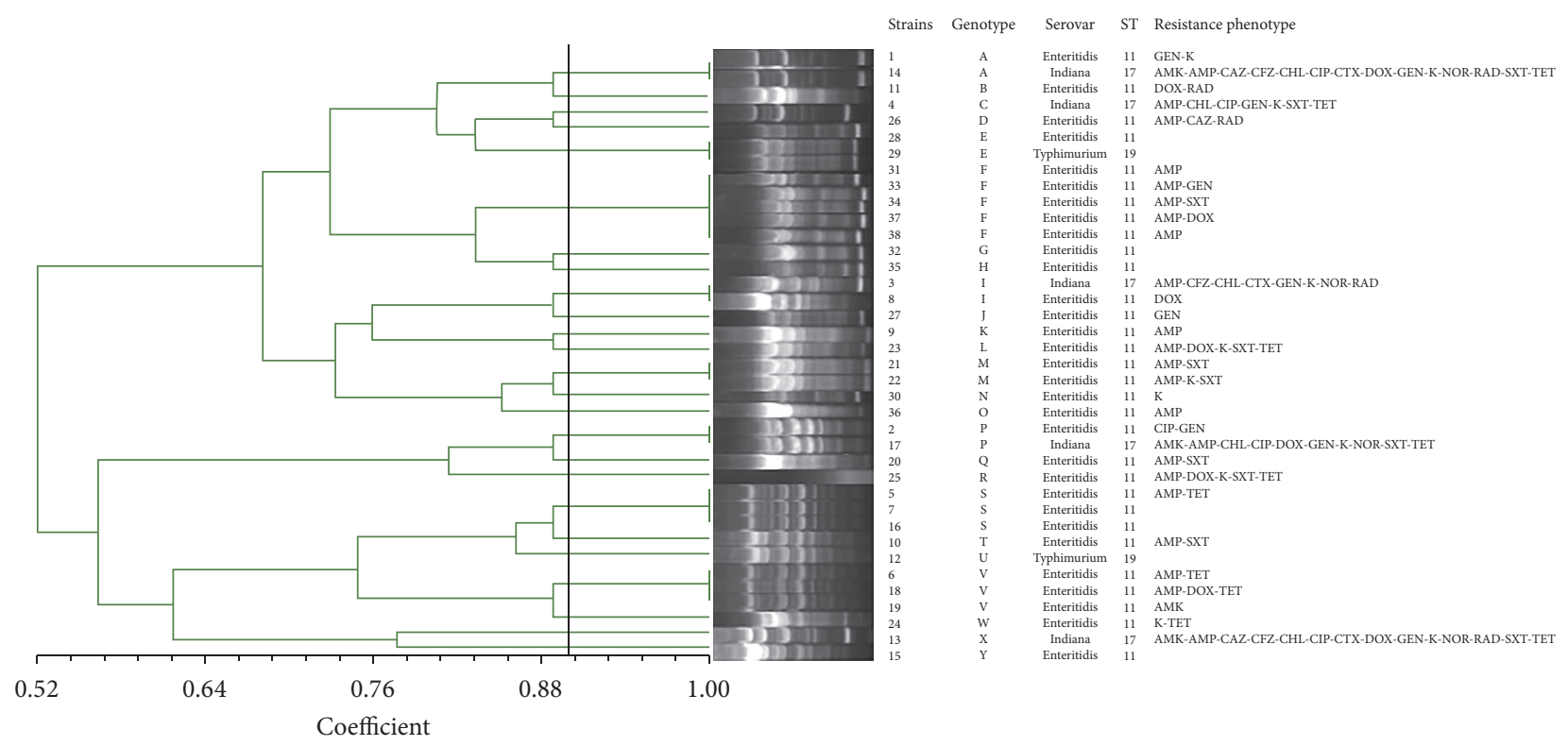

FIGURE 1: Dendrogram of Salmonella isolates from free-range chickens by ERIC-PCR.

TABLE 2: Antibiotic resistance rates of Salmonella isolated from three free-range chicken farms.

\begin{tabular}{lc}
\hline Antimicrobials & Antibiotics resistance rates N (\%) \\
\hline Amikacin & $4(10.5 \%)$ \\
Ampicillin & $22(57.9 \%)$ \\
Cefazolin & $3(7.9 \%)$ \\
Cefotaxime & $3(7.9 \%)$ \\
Ceftazidime & $3(7.9 \%)$ \\
Cephradine & $5(13.2 \%)$ \\
Chloramphenicol & $5(13.2 \%)$ \\
Ciprofloxacin & $5(13.2 \%)$ \\
Co-trimoxazole & $11(29.0 \%)$ \\
Doxycycline & $9(23.7 \%)$ \\
Gentamicin & $9(23.7 \%)$ \\
Kanamycin & $11(29.0 \%)$ \\
Norfloxacin & $4(10.5 \%)$ \\
Tetracycline & $10(26.3 \%)$ \\
\hline
\end{tabular}

and ceftazidime, $7.9 \%$ each (3/38). Ten of thirty-eight isolates (26.3\%) were MDR (Tables 2 and 3).

3.4. ERIC-PCR Analysis. The 38 Salmonella isolates could be divided into 25 genotypes by ERIC-PCR. Genetic similarity ranged from $52 \%$ to $100 \%$. Of note, the genetic similarity of the following isolates was 100\%: numbers 1 and 14; numbers 2 and 17; numbers 3 and 8 ; numbers 21 and 22; numbers 28 and 29; numbers 5, 7, and 16; numbers 6, 18, and 19; and numbers 31, 33, 34, 37, and 38 (Figure 1).

3.5. MLST. The thirty-eight Salmonella isolates were classified into three ST types. ST11 was the highest isolate rate $(31 / 38,81.6 \%)$, which belongs to the clonal complex 258
(CC258), followed by ST17 (5/38, 13.2\%) and ST19 (2/3, 85.3\%) (Figure 1).

\section{Discussion}

Salmonella is an important pathogen of great importance in public health. Poultry are considered to be important carrier of Salmonella [17, 18]. Numerous studies have been conducted on Salmonella isolated from intensive breeding chicken farms, slaughter houses, and chicken meats [2, 1921], but little information is available on free-range chickens. In the present study, the overall isolate rate of Salmonella in three free-range chickens was $12.7 \%$, which was lower than that in intensive chicken farms in China (>35\%) [22].

Numerous Salmonella serotypes are pathogenic, including S. enterica serovar Enteritidis and S. enterica serovar Typhimurium [23]. The most common serotype identified in the present study was S. enterica serovar Enteritidis (81.6\%). It was consistent with investigation results from the intensively managed chicken farms in the Henan and Sichuan areas of China $[24,25]$. But the most common isolated Salmonella from the intensively managed chicken farms in Cambodia, Vietnam, and South Korea were S. enterica serovar Anatum, S. enterica serovar Infantis, and S. enterica serovar Hadar, respectively $[17,26,27]$. The difference of the Salmonella serotype distribution may mainly be related with area differences.

Compared with the resistant rate of Salmonella from intensive poultry farms in Shandong province, China, in 2012, in the present study, the resistant rate of these 38 Salmonella isolates against ampicillin, kanamycin, co-trimoxazole, and tetracycline was greatly lower (36.1-72.3\% versus $26.3-57.9 \%$ ) [28]. This apparent difference may be attributable to less drug use in free-range chickens. Of note, Salmonella isolates in this study were relatively sensitive to cephalosporin antibiotics 
TABLE 3: Antimicrobial resistance patterns of Salmonella isolated from three free-range chicken farms.

\begin{tabular}{|c|c|c|c|c|}
\hline \multirow{2}{*}{ Resistant patterns } & \multicolumn{4}{|c|}{ Number of resistant isolates } \\
\hline & Enteritidis & Indiana & Typhimurium & Total \\
\hline $\begin{array}{l}\text { AMK-AMP-CAZ-CFZ-CHL-CIP-CTX-DOX-GEN-K-NOR-RAD-SXT-TET } \\
\end{array}$ & 0 & 2 & 0 & 2 \\
\hline AMK-AMP-CHL-CIP-DOX-GEN-NOR-K-SXT-TET & 0 & 1 & 0 & 1 \\
\hline AMK-CFZ-CHL-CTX-GEN-K-NOR-RAD & 0 & 1 & 0 & 1 \\
\hline AMP-CHL-CIP-GEN-K-SXT-TET & 0 & 1 & 0 & 1 \\
\hline AMP-DOX-K-SXT-TET & 2 & 0 & 0 & 2 \\
\hline AMP-CAZ-RAD & 1 & 0 & 0 & 1 \\
\hline AMP-DOX-TET & 1 & 0 & 0 & 1 \\
\hline AMP-K-SXT & 1 & 0 & 0 & 1 \\
\hline AMP-DOX & 1 & 0 & 0 & 1 \\
\hline AMP-GEN & 1 & 0 & 0 & 1 \\
\hline AMP-SXT & 4 & 0 & 0 & 4 \\
\hline AMP-TET & 2 & 0 & 0 & 2 \\
\hline CIP-GEN & 1 & 0 & 0 & 1 \\
\hline GEN-K & 1 & 0 & 0 & 1 \\
\hline K-TET & 1 & 0 & 0 & 1 \\
\hline DOX-RAD & 1 & 0 & 0 & 1 \\
\hline AMK & 1 & 0 & 0 & 1 \\
\hline AMP & 4 & 0 & 0 & 4 \\
\hline GEN & 1 & 0 & 0 & 1 \\
\hline DOX & 1 & 0 & 0 & 1 \\
\hline $\mathrm{K}$ & 1 & 0 & 0 & 1 \\
\hline
\end{tabular}

(resistance rate, 7.9-13.2\%), and these values are lower than the recorded values for cephalosporin antibiotics resistance in chickens raised in intensively managed farms in Shandong province, China (resistance rate, about $42 \%$ in 2012) [28]. Our findings showed that $26.3 \%$ of Salmonella isolates were MDR. This was lower than that in intensive poultry farms of Henan (46.0\%) province in China [24]. Of note, in this study 2 out of $5 \mathrm{~S}$. enterica serovar Indiana isolates were resistant to 14 antibiotics, and they were not only resistant to streptomycin and tetracycline but also resistant to chloramphenicol, fluoroquinolones, and cephalosporin antibiotics. The antibiotic resistance difference of Salmonella isolated from three freerange chicken farms may be due to the fact that the use of antibiotics in different regions is quite different and the distribution of the antibiotics in the environments, such as waters and soils, has a great effect on drug resistance of Salmonella.

The result of ERIC-PCR showed that the 38 Salmonella isolates were classified into 25 gene types, which indicates that the sources of Salmonella isolates in these flocks were diverse. Compared with Salmonella isolates from Laiwu, the isolates from Jinan and Linyi had lower genetic similarity. The difference should be further studied. The fact that isolates from Laiwu had higher genetic similarity may be associated with clonal spread of Salmonella. Thirty-eight Salmonella isolates were classified into 3 ST types (ST11, ST17, and ST19). All three types have also been isolated from human samples [29-31], suggesting that these Salmonella could be spread between human beings and chickens via food chain and threaten the human health.

\section{Conclusions}

Collectively, Salmonella isolated from free-ranging chickens showed relatively lower resistance rates than those raised in intensively managed chicken farms of China. These results also revealed that free-ranging chickens may serve as a potential reservoir for antibiotic resistance Salmonella, thereby posing a threat to public health.

\section{Competing Interests}

The authors declare no competing interests.

\section{Authors' Contributions}

Xiaonan Zhao and Yanxia Gao contributed equally to this work.

\section{Acknowledgments}

The authors acknowledge the financial support from the Special Grant of Innovation Team of Shandong Province (SDAIT-13-011-11) and the Development Plan of Science and Technology of Shandong Province (no. 2014GSF119024).

\section{References}

[1] E. Scallan, R. M. Hoekstra, F. J. Angulo et al., "Foodborne illness acquired in the United States-Major pathogens," Emerging Infectious Diseases, vol. 17, no. 1, pp. 7-15, 2011. 
[2] M. D. Kirk, S. M. Pires, R. E. Black et al., "World Health Organization estimates of the global and regional disease burden of 22 food-borne bacterial, protozoal, and viral diseases, 2010: a data synthesis," PLoS Medicine, vol. 12, Article ID e1001921, 2015.

[3] S. Cui, J. Li, Z. Sun et al., "Characterization of Salmonella enterica isolates from infants and toddlers in Wuhan, China," Journal of Antimicrobial Chemotherapy, vol. 63, no. 1, pp. 87-94, 2009.

[4] Y. Li, X. Xie, X. Xu et al., "Nontyphoidal Salmonella infection in children with acute gastroenteritis: prevalence, serotypes, and antimicrobial resistance in Shanghai, China," Foodborne Pathogens and Disease, vol. 11, no. 3, pp. 200-206, 2014.

[5] Z. Liang, B. Ke, X. Deng et al., "Serotypes, seasonal trends, and antibiotic resistance of non-typhoidal from human patients in Guangdong Province, China, 2009-2012," BMC Infectious Diseases, vol. 15, no. 1, article 53, 2015.

[6] D. Shao, Z. Shi, J. Wei, and Z. Ma, "A brief review of food-borne zoonoses in China," Epidemiology and Infection, vol. 139, pp. 1497-1504, 2011.

[7] W. H. Zhang, X. Y. Lin, L. Xu et al., "CTX-M-27 producing salmonella enterica serotypes typhimurium and indiana are prevalent among food-producing animals in China," Frontiers in Microbiology, vol. 7, article 436, 2016.

[8] J.-W. Chon, H.-I. Jung, M. Kuk, Y.-J. Kim, K.-H. Seo, and S.K. Kim, "High occurrence of extended-spectrum $\beta$-lactamaseproducing salmonella in broiler carcasses from poultry slaughterhouses in South Korea," Foodborne Pathogens and Disease, vol. 12, no. 3, pp. 190-196, 2015.

[9] T. Noda, K. Murakami, Y. Etoh et al., "Increase in resistance to extended-spectrum cephalosporins in Salmonella isolated from retail chicken products in Japan," PLoS ONE, vol. 10, no. 2, Article ID e0116927, 2015.

[10] S. Bonardi, I. Alpigiani, I. Bruini et al., "Detection of Salmonella enterica in pigs at slaughter and comparison with human isolates in Italy," International Journal of Food Microbiology, vol. 218, pp. 44-50, 2016.

[11] B. Malorny, J. Hoorfar, M. Hugas et al., "Interlaboratory diagnostic accuracy of a Salmonella specific PCR-based method," International Journal of Food Microbiology, vol. 89, no. 2-3, pp. 241-249, 2003.

[12] P. A. D. Grimont and F. X. Weill, Antigenic Formulae of the Salmonella Serovars, WHO collaborating Centre for Reference and Research on Salmonella, Institut Pasteur, Paris, France, 9th edition, 2007.

[13] Clinical and Laboratory Standards Institute (CLSI), "Performance standards for antimicrobial susceptibility testing," Twentieth-Third Informational Supplement M100-S23, Clinical and Laboratory Standards Institute (CLSI), Wayne, Pa, USA, 2013.

[14] A.-P. Magiorakos, A. Srinivasan, R. B. Carey et al., "Multidrugresistant, extensively drug-resistant and pandrug-resistant bacteria: an international expert proposal for interim standard definitions for acquired resistance," Clinical Microbiology and Infection, vol. 18, no. 3, pp. 268-281, 2012.

[15] S. Li, W. Song, Y. Zhou, Y. Tang, Y. Gao, and Z. Miao, "Spread of extended-spectrum beta-lactamase-producing Escherichia coli from a swine farm to the receiving river," Environmental Science and Pollution Research, vol. 22, no. 17, pp. 13033-13037, 2015.
[16] T. Papadopoulos, E. Petridou, A. Zdragas et al., "Comparative study of all Salmonella enterica serovar Enteritidis strains isolated from food and food animals in Greece from 2008 to 2010 with clinical isolates," European Journal of Clinical Microbiology and Infectious Diseases, vol. 35, pp. 741-746, 2016.

[17] T. H. Thai and R. Yamaguchi, "Molecular characterization of antibiotic-resistant Salmonella isolates from retail meat from markets in Northern Vietnam," Journal of Food Protection, vol. 75, no. 9, pp. 1709-1714, 2012.

[18] W. Yamazaki, R. Uemura, S. Sekiguchi et al., "Campylobacter and Salmonella are prevalent in chicken farms in Kyushu, Japan: results of a 2-year distribution and circulation dynamics audit," Journal of Applied Microbiology, vol. 120, no. 6, pp. 1711-1722, 2016.

[19] B. Yang, D. Qu, X. Zhang et al., "Prevalence and characterization of Salmonella serovars in retail meats of marketplace in Shaanxi, China," International Journal of Food Microbiology, vol. 141, no. $1-2$, pp. 63-72, 2010

[20] M. Aslam, S. Checkley, B. Avery et al., "Phenotypic and genetic characterization of antimicrobial resistance in Salmonella serovars isolated from retail meats in Alberta, Canada," Food Microbiology, vol. 32, no. 1, pp. 110-117, 2012.

[21] D. H. Bae, H. K. Dessie, H. J. Baek, S. G. Kim, H. S. Lee, and Y. J. Lee, "Prevalence and characteristics of Salmonella spp. Isolated from poultry slaughterhouses in Korea," Journal of Veterinary Medical Science, vol. 75, no. 9, pp. 1193-1200, 2013.

[22] Y. Lu, C.-M. Wu, G.-J. Wu et al., "Prevalence of antimicrobial resistance among Salmonella isolates from chicken in China," Foodborne Pathogens and Disease, vol. 8, no. 1, pp. 45-53, 2011.

[23] T. Sivaramalingam, D. L. Pearl, S. A. McEwen, D. Ojkic, and M. T. Guerin, "A temporal study of Salmonella serovars from fluff samples from poultry breeder hatcheries in Ontario between 1998 and 2008," Canadian Journal of Veterinary Research, vol. 77, no. 1, pp. 12-23, 2013.

[24] L. Bai, R. Lan, X. Zhang et al., "Prevalence of salmonella isolates from chicken and pig slaughterhouses and emergence of ciprofloxacin and cefotaxime co-resistant S. enterica serovar Indiana in Henan, China," PLoS ONE, vol. 10, no. 12, Article ID e0144532, 2015.

[25] R. Li, J. Lai, Y. Wang et al., "Prevalence and characterization of Salmonella species isolated from pigs, ducks and chickens in Sichuan Province, China," International Journal of Food Microbiology, vol. 163, no. 1, pp. 14-18, 2013.

[26] K. S. Lay, Y. Vuthy, P. Song, K. Phol, and J. L. Sarthou, "Prevalence, numbers and antimicrobial susceptibilities of Salmonella serovars and Campylobacter spp. in retail poultry in Phnom Penh, Cambodia," Journal of Veterinary Medical Science, vol. 73, no. 3, pp. 325-329, 2011.

[27] S.-W. Choi, J.-S. Ha, B.-Y. Kim et al., "Prevalence and characterization of Salmonella species in entire steps of a single integrated broiler supply chain in Korea," Poultry Science, vol. 93, no. 5, pp. 1251-1257, 2014.

[28] J. Lai, C. Wu, C. Wu et al., "Serotype distribution and antibiotic resistance of Salmonella in food-producing animals in Shandong province of China, 2009 and 2012," International Journal of Food Microbiology, vol. 180, pp. 30-38, 2014.

[29] R. Elgroud, S. A. Granier, M. Marault et al., "Contribution of avian Salmonella enterica isolates to human salmonellosis cases in constantine (Algeria)," BioMed Research International, vol. 2015, Article ID 352029, 8 pages, 2015. 
[30] X. Kuang, H. Hao, M. Dai et al., "Antimicrobial susceptibility and molecular typing of salmonella agona isolated from humans and other sources," Foodborne Pathogens and Disease, vol. 11, no. 11, pp. 844-849, 2014.

[31] X. Yang, D. Kuang, J. Meng et al., "Antimicrobial resistance and molecular typing of Salmonella stanley isolated from humans, foods, and environment," Foodborne Pathogens and Disease, vol. 12, no. 12, pp. 945-949, 2015. 


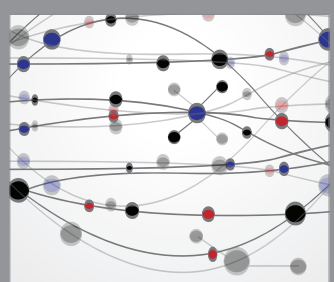

The Scientific World Journal
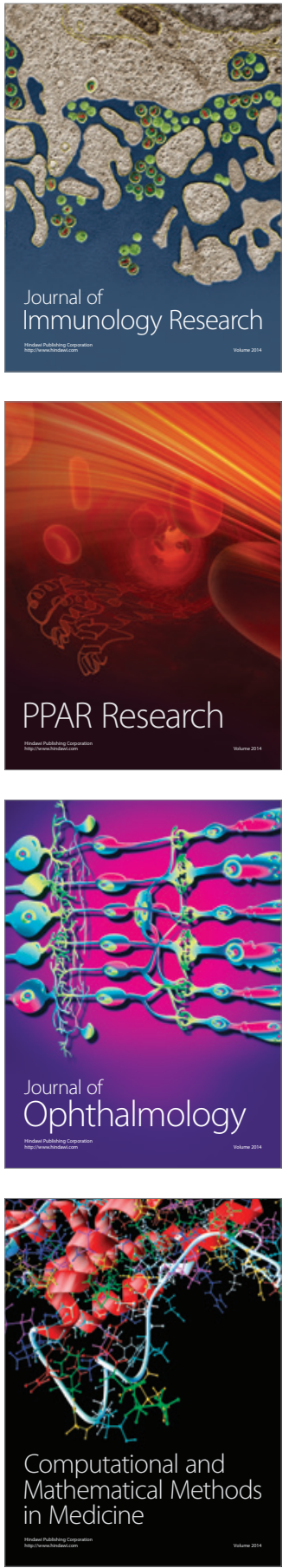

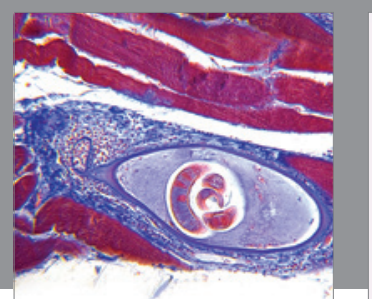

Gastroenterology Research and Practice

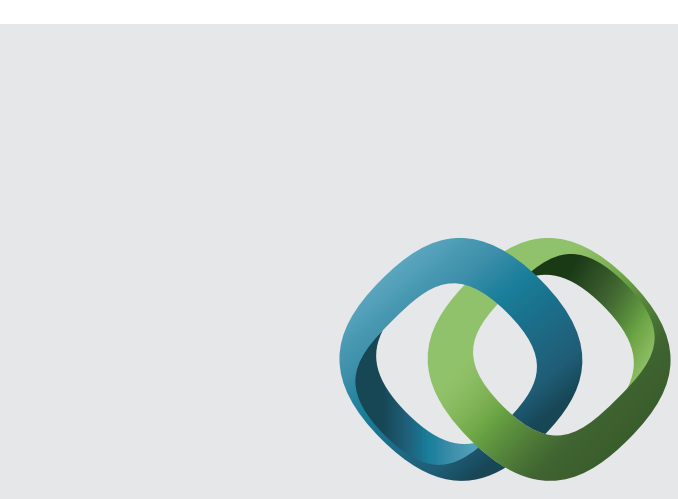

\section{Hindawi}

Submit your manuscripts at

http://www.hindawi.com
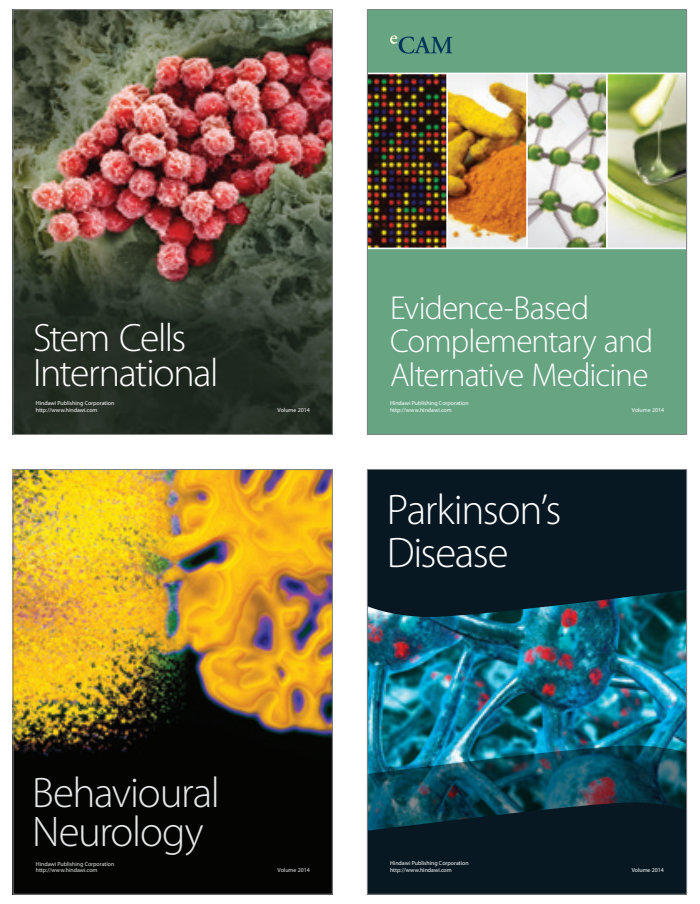
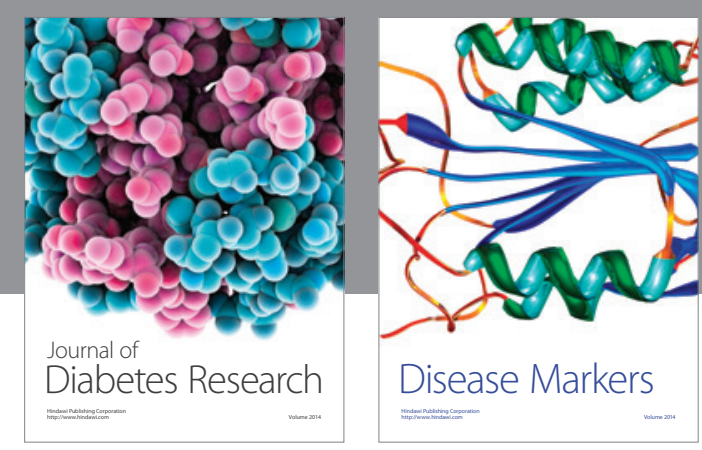

Disease Markers
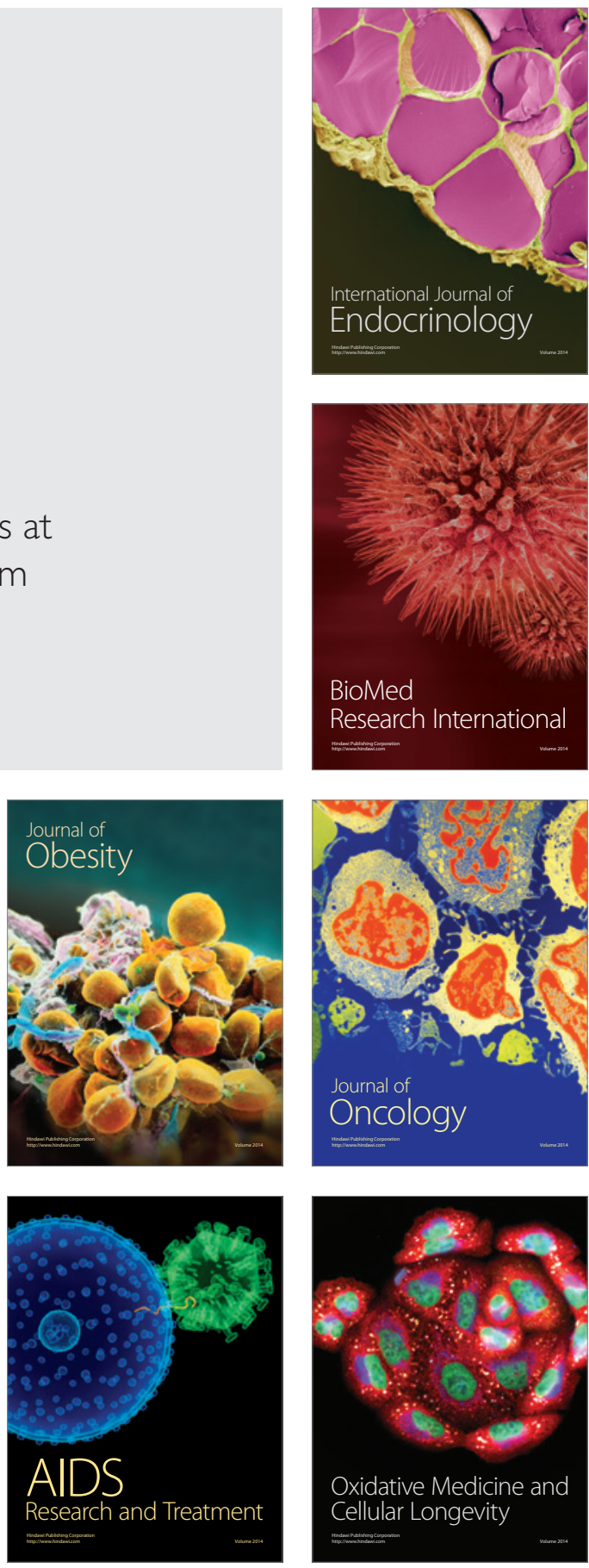